\title{
MODELAGEM DE EQUAÇÕES ESTRUTURAIS APLICADA A UM ESTUDO DA PERCEPÇÃO DE MILITARES SOBRE A ESCASSEZ DE RECURSOS DESTINADOS PELO GOVERNO ÀS FORÇAS ARMADAS
}

\author{
Giovani Glaucio de Oliveira Costa \\ Universidade Federal Rural do Rio de Janeiro \\ giovaniglaucio@ufrrj.br
}

\section{Resumo}

O Ministério da Defesa vem alertando às autoridades do governo federal, em diversas instâncias, que os recursos destinados às Forças Armadas são insuficientes para suprir as necessidades de custeio, defesa, desenvolvimento de ensino e pesquisa. Este artigo objetiva, através da modelagem de equações estruturais, identificar constructos latentes, relações causais, que possam explicar de forma essencial, a percepção de militares ativos a cerca de recursos escassos no desenvolvimento de suas atividades diárias. O estudo visa buscar apoio dos militares ativos, que vivenciam a realidade militar em seu dia a dia, aos argumentos do Ministério da Defesa de que o orçamento é insuficiente para as necessidades mínimas do setor. Acredita-se que a relevância deste estudo está na produção de conhecimento que pudesse cientificamente fundamentar as argumentações dos representantes das armas de que a área da defesa requer maiores recursos para o bem promover a defesa e a proteção das fronteiras de nosso país e assim garantir sempre a soberania nacional.

Palavras-chaves: forças armadas, escassez de recursos, percepção de militares, análise fatorial exploratória, modelagem de equações estruturais.

\begin{abstract}
The Ministry of defense has been alerting the authorities of the federal Government, in several instances, that the resources allocated to the armed forces are insufficient to meet the needs of costing, defense, development of teaching and research. This article aims, through structural equation modeling, identifying latent constructs, causal relations, which may explain so essential, the perception of active military, what sectors of the armed forces are actually in lag of resources by the Government. The study aims to seek support from the military assets, to experience the military reality in their daily lives, the Defense Ministry's arguments that the budget is insufficient for the minimum needs of the sector. It is believed that the relevance of this study is in the production of knowledge that could be scientifically substantiate the arguments of representatives of arms that the defense area requires greater resources for promote the defense and protection of the borders of our country and so ensure the national sovereignty.
\end{abstract}

Key-words: military, resource scarcity, perception of military, exploratory factor analysis, structural equation modeling. 


\section{1-INTRODUÇÃO}

As Forças Armadas do Brasil são divididas em 3 ramos: Marinha do Brasil, Exército Brasileiro e Força Aérea Brasileira. A Polícia Militar do Brasil (polícia estadual) ao lado do Corpo de Bombeiros Militar são descritos como uma força auxiliar e de reserva do Exército. Todos os ramos militares são parte do Ministério da Defesa.

A Marinha do Brasil que é o mais antigo das Forças Armadas brasileiras inclui o Corpo de Fuzileiros Navais do Brasil e da Aviação Naval Brasileira. O Brasil tem a mais poderosa força militar da América do Sul, assim como cada um de seus ramos militares.

Uma nota técnica elaborada pela área militar em 2013, encaminhada à Comissão de Relações Exteriores e Defesa Nacional do Senado, mostra um cenário preocupante para as Forças Armadas brasileiras em 2014. Um documento, que serviu de subsídio para uma audiência pública em novembro de 2013, apontou uma diferença de $\mathrm{R} \$ 13,65$ bilhões entre o que as três armas consideram como "necessidades mínimas" e o que estava previsto no orçamento de 2014. Os militares pediam $\mathrm{R} \$ 29,828$ bulhões, mas os recursos com os quais poderiam contar somariam somente $\mathrm{R} \$ 16,178$ bilhões.

O Exército, na época, alertou, no documento, que entre as consequências para a falta de recursos estaria o enfraquecimento da segurança na faixa da fronteira, especialmente na Amazônia e no Centro-oeste. O Exército aponta, também, problemas de aquisição de munição de todos os calibres e também se diz preocupado com os sistemas de defesa antiaéreo e cibernético.

A Marinha afirmou, até então, que diversos projetos, entre eles a construção de navios-patrulha de 500 toneladas para prover segurança na área do pré-sal e a re-construção da Estação Antártica, estão comprometidos. Segundo a Marinha, há uma forte demanda reprimida na manutenção da Esquadra, o que causa aceleração da defasagem tecnológica, perda de mão de obra qualificada pela queda da qualidade do ensino e aumento significativos nos custos de operação.

A Aeronáutica, responsável pela proteção de um espaço aéreo que vai além do próprio território brasileiro, uma dimensão de 22 milhões de quilômetros quadrados, elenca como prioridades que não podem ser desprezadas o Veículo Lançador de Satélites (VLS), a compra de cargueiros para a substituição imediata das aeronaves KC137(Boeing 707 adaptado para fins militares), a aquisição de 28 aeronaves KC-390 e a aquisição da caça F-5. A aeronáutica informa que estão paradas 346 de 624 aeronaves com capacidade de operação.

Os recursos de Orçamento da União que vêm sendo destinados nos últimos anos à Defesa não estão sendo suficientes para atender sequer ao custeio das Forças Armadas, a exemplo da manutenção de equipamentos, e muitos menos aos projetos contemplados na Estratégia Nacional de Defesa.

O senso comum associa às Forças Armadas apenas ao combate e à defesa do país em termos de conflito, ignorando o trabalho dessas mesmas forças em tempos de paz, na proteção de interesses nacionais e no dia a dia do brasileiro. O Ministério da Defesa declara que é 
necessário que os gastos do Brasil no setor aumentem de 1,5\% para $2 \%$ do Produto Interno Bruto (PIB). A expansão poderia se dá em um período de dez anos.

De acordo coma a Defesa, apesar das dificuldades que também atingem outros ministérios, houve aumento substancial de recursos para a pasta na última década: a verba para custeio e investimentos subiu de R \$ 4,6 bilhões em 2003 para R \$ 19,6 bilhões (número divergente do da nota técnica elaborada pela área militar em 2013), como previsto no projeto de lei orçamentária para 2014. Isso significa uma expansão de $326 \%$.

Este artigo objetiva, através da Modelagem de Equações Estruturais, identificarem constructos latentes, relações causais, que possam explicar de forma essencial, na percepção de militares ativos, que setores das forças armadas estão realmente em defasagem de recursos destinados pelo governo.

O estudo visa buscar apoio dos militares ativos, que vivenciam a realidade militar em seu dia a dia, aos argumentos do Ministério da Defesa de que o orçamento é insuficiente para as necessidades mínimas do setor. A pesquisa pode ajudar no entendimento de que setores da defesa estão na percepção diária dos militares em defasagem de recursos destinados pelo governo e assim ratificar o alerta de que o cenário assim configurado deve ser repensado e revisto através de tomadas de decisão do poder público.

\section{2- METODOLOGIAS DA PESQUISA}

Foi realizada uma pesquisa de opinião junto a 40 militares do Brasil das três Forças Armadas. A pesquisa foi realizada em áreas militares e definida como um levantamento por amostragem não probabilística por quotas, com erro de amostragem de 15,5\%, para mais ou para menos. Cada militar teria que dá uma nota de 1 a 7 sobre sua percepção da carência de recursos em ensino, custeio, pesquisa e defesa.

A análise de dados consistirá na identificação de constructos ou variáveis latentes causais do comportamento das percepções Ensino, Custeio, Pesquisa e Defesa através da Modelagem de Equações Estruturais.

Espera-se que com o uso da referida técnica se consiga confirmar a teoria sobre que fatores latentes, escalonados por ordem de importância, são percebidos pelos militares como a essência de carência de recursos na área de defesa.

Para realizar a análise pretendida, serão realizadas duas etapas:

$\left.1^{a}\right)$ Uma análise fatorial exploratória que visa identificar os fatores latentes;

$\left.2^{a}\right)$ Uma análise fatorial confirmatória, através da modelagem de equações estruturais, que visará confirmar a estrutura de relações causais teorizadas pelos resultados da análise fatorial exploratória, realizada na primeira etapa. 


\section{3-DESENVOLVIMENTO DA ANÁLISE FATORIAL EXPLORATÓRIA}

A análise fatorial exploratória visará criar uma teoria que fundamente as relações causais do comportamento das percepções dos militares quanto à falta de recursos nas forças armadas. Os parágrafos a seguir descreverão o desenvolvimento da referida técnica multivariada.

Tabela 1

Matriz de Correlações

Correlation Matrix

\begin{tabular}{|ll|r|r|r|r|}
\hline & & \multicolumn{1}{|c|}{ Ensino } & \multicolumn{1}{c|}{ Custeio } & \multicolumn{1}{c|}{ Pesquisa } & \multicolumn{1}{c|}{ Defesa } \\
\hline Correlation & Ensino & 1.000 & -.428 & .765 & -.345 \\
& Custeio & -.428 & 1.000 & -.356 & .791 \\
& Pesquisa & .765 & -.356 & 1.000 & -.408 \\
& Defesa & -.345 & .791 & -.408 & 1.000 \\
\hline Sig. (1-tailed) & Ensino & & .003 & .000 & .015 \\
& Custeio & .003 & & .012 & .000 \\
& Pesquisa & .000 & .012 & & .004 \\
& Defesa & .015 & .000 & .004 & \\
\hline
\end{tabular}

Observado a tabela 1 , verificamos correlações significativas, o que implica que as variáveis envolvidas deverão estar sob influência de fatores latentes. Muitas das correlações estão dentro do limite satisfatório para que a multicolinearidade não seja problema.

Tabela 2

Teste de Esfericidade de Bartlett

Teste KMO

KMO and Bartlett's Test

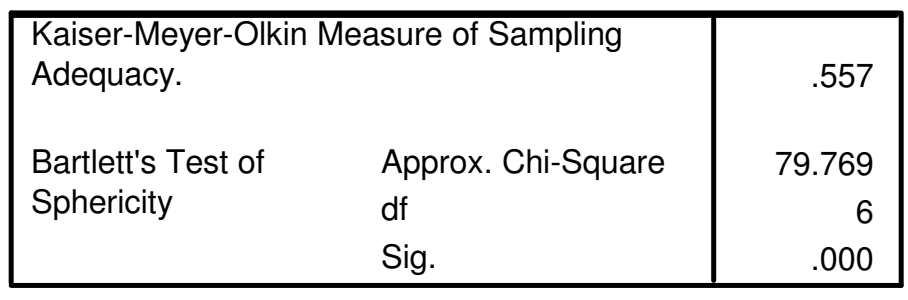

A tabela 2 informa que o valor-p do teste de esfericidade de Bartlett é 0,000, o que implica em rejeitar a hipótese nula de que a matriz de correlação é a matriz identidade. Existe correlação estatisticamente significante entre as variáveis. $\mathrm{O}$ valor do teste de KMO foi 0,557. Este resultado indica que o uso do método das componentes principais como indicadoras de variáveis latentes é aceitável. 
Tabela 3

Comunalidades

Communalities
\begin{tabular}{|l|r|r|}
\hline & \multicolumn{1}{|c|}{ Initial } & Extraction \\
\hline Ensino & 1.000 & .883 \\
Custeio & 1.000 & .894 \\
Pesquisa & 1.000 & .882 \\
Defesa & 1.000 & .897 \\
\hline
\end{tabular}

Extraction Method: Principal Component Analysis.

A tabela 3 informa valores aceitáveis para as comunalidades. Cada variável observada compartilha de correlações altas com as outras. Como as correlações estão próximas de 1, pode-se evidenciar que existem variáveis latentes que explicam quase toda a variação das percepções de militares quanto aos recursos escassos na forças armadas.

Tabela 4

Total de Variância Explicada

\section{Total Variance Explained}

\begin{tabular}{|l|r|r|r|r|r|r|r|r|r|}
\hline & \multicolumn{3}{|c|}{ Initial Eigenvalues } & \multicolumn{4}{c|}{ action Sums of Squared Loaditation Sums of Squared Loadin } \\
\cline { 2 - 9 } Compone & \multicolumn{1}{|c|}{ Total } & of Varianqumulative & \multicolumn{2}{|c|}{ Total } & of Variancumulative & Total & of Variandumulative \\
\hline 1 & 2.546 & 63.658 & 63.658 & 2.546 & 63.658 & 63.658 & 1.789 & 44.731 & 44.731 \\
2 & 1.009 & 25.234 & 88.892 & 1.009 & 25.234 & 88.892 & 1.766 & 44.161 & 88.892 \\
3 & .291 & 7.275 & 96.167 & & & & & & \\
4 & .153 & 3.833 & 100.000 & & & & & & \\
\hline
\end{tabular}

Extraction Method: Principal Component Analysis.

Os dois primeiros fatores retém juntos $\mathbf{8 9 \%}$ da variância total, o que sugere que os dois primeiros fatores explicam substancialmente as inter-relações das percepções de militares quanto aos recursos escassos na forças armadas. O scree plot, gráfico dos autovalores, apresentado no gráfico 1, revela valores significativos até o fator 2 . Em seguida, ocorre uma queda, o que ratifica o uso de somente dos dois primeiros fatores para variáveis latentes. 


\section{Gráfico 1}

Scree Plot

\section{Scree Plot}

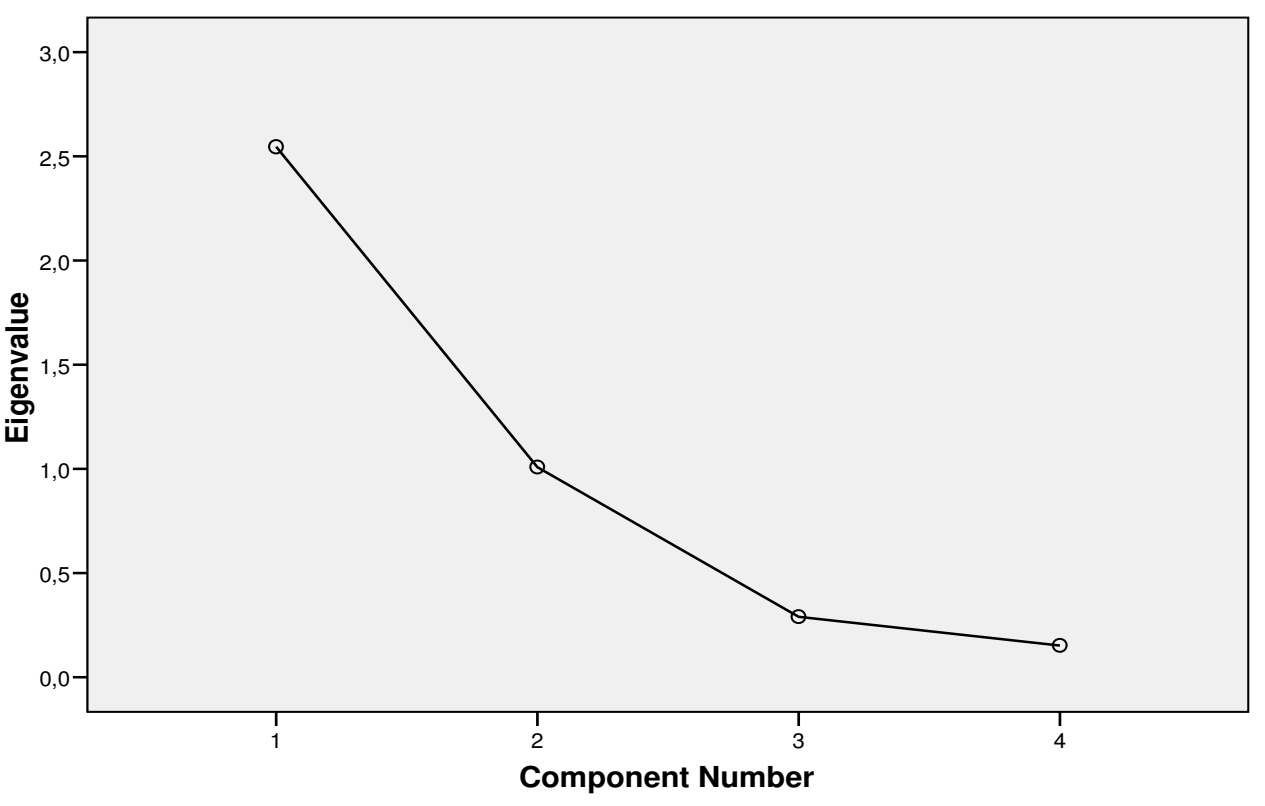

Tabela 5

Matriz de Fatores

\begin{tabular}{|l|r|r|}
\multicolumn{2}{c|}{ Component Matrix } \\
\hline \multirow{2}{*}{} & \multicolumn{2}{|c|}{ Component } \\
\cline { 2 - 3 } & 1 & \multicolumn{1}{c|}{2} \\
\hline Ensino & -.793 & .505 \\
Custeio & .810 & .489 \\
Pesquisa & -.790 & .508 \\
Defesa & .799 & .508 \\
\hline
\end{tabular}

Extraction Method: Principal Component Analysis.

a. 2 components extracted.

A matriz de fatores ainda não traduz com segurança a importância de cada variável em cada fator. Ter-se-á que observar a matriz rotada de fatores. 
Tabela 6

Matriz Rotada de Fatores

Rotated Component Matrix

\begin{tabular}{|l|r|r|}
\hline \multirow{2}{*}{} & \multicolumn{2}{|c|}{ Component } \\
\cline { 2 - 3 } & \multicolumn{1}{|c|}{1} & \multicolumn{1}{c|}{2} \\
\hline Ensino & -.210 & .916 \\
Custeio & .920 & -.220 \\
Pesquisa & -.206 & .916 \\
Defesa & .926 & -.199 \\
\hline
\end{tabular}

Extraction Method: Principal Component Analysis.

Rotation Method: Varimax with Kaiser Normalization.

a. Rotation converged in 3 iterations.

A matriz de fatores rotada já elucida as variáveis mais importantes para cada variável latente detectada. O fator 1 é discriminado pelas variáveis Custeio e Defesa. $O$ fator 2 é representado pelas variáveis Ensino e Pesquisa. Portanto, a análise fatorial exploratória detectou dois fatores causais, isto é, o que intrinsecamente os militares percebem como carentes de recursos nas armas: "Fator Necessidades Minimas" e "Fator Necessidades de Aperfeiçoamento". O "Fator Necessidades Minimas" responde por 45\% das percepções dos militares de falta de recursos e o "Fator Necessidades de Aperfeiçoamento", por 44\%. Essencialmente, as percepções de militares quanto aos recursos escassos nas forças armadas são ditados por estes dois constructos latentes. Este é o suporte teórico que se fundamentará a Modelagem de Equações Estruturais formulada neste artigo. A próxima etapa deste estudo é promover uma modelagem de equações estruturais e realizar uma análise fatorial confirmatória dos resultados.

\section{4-DESENVOLVIMENTO DA ANÁLISE DE EQUAÇÕES ESTRUTURAIS}

A próxima etapa da análise é confirmar os resultados até então encontrados pela modelagem de equações estruturais. Esta etapa consiste na análise fatorial confirmatória. As hipóteses ou teoria para a modelagem são que existem dois constructos latentes que explicam de maneira considerável as percepções de militares quanto aos recursos escassos na forças armadas: "Fator Necessidades Mínimas" e "Fator Necessidades de Aperfeiçoamento". Os indicadores da variável latente "Fator Necessidades Mínimas" são "Custeio" e "Defesa". Os indicadores da variável latente "Fator Necessidades de Aperfeiçoamento" são "Ensino" $e$ "Pesquisa". A teoria termina com a suposição de colinearidade entre os constructos do modelo. Será realizada a modelagem de equações estruturais no AMOS. Os resultados são apresentados a seguir. O diagrama de caminhos da análise foi obtida junto ao $A M O S$ : 


\section{Gráfico 2}

Diagrama de Caminhos de Relações de Percepções de Militares quanto a Falta de Recursos das Forças Armadas

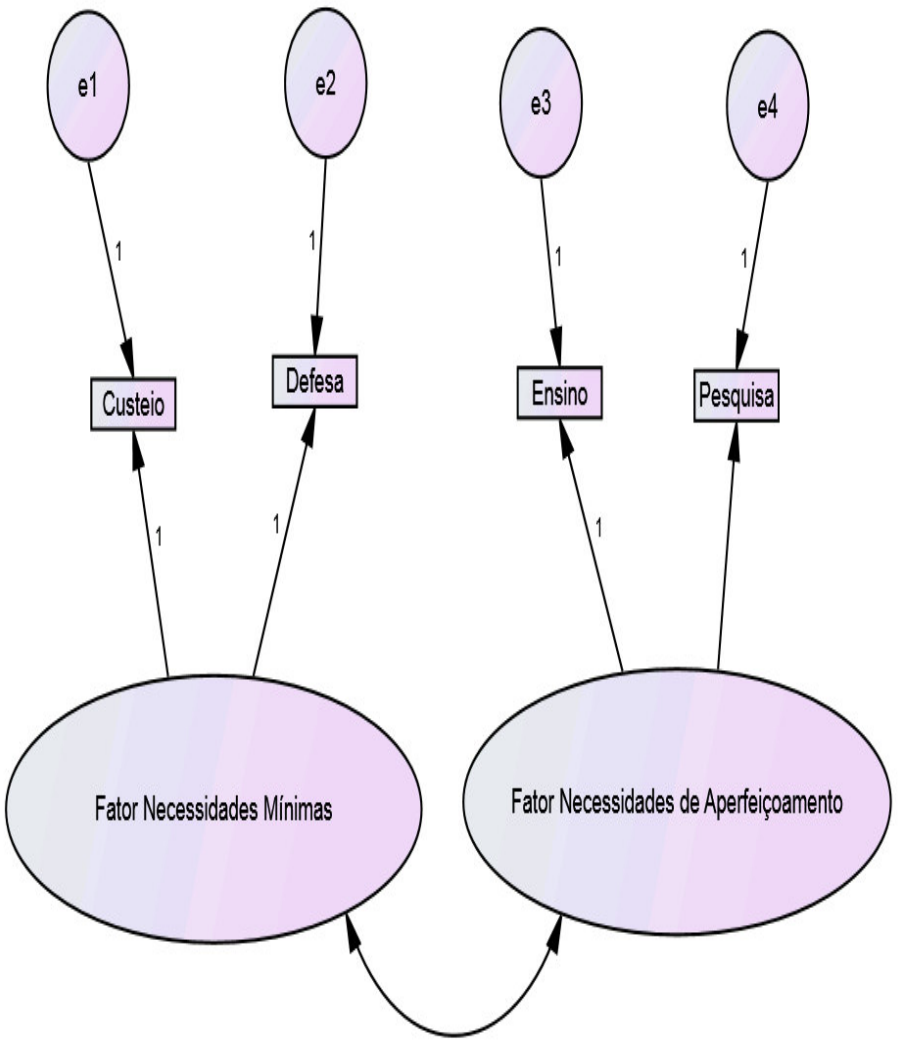

As variáveis latentes Fator de Necessidades Mínimas e Fator Necessidades de Aperfeiçoamento são os constructos ou os conceitos abstratos latentes e por hipótese com colinearidade. Os indicadores do Fator de Necessidades Mínimas são as variáveis observadas "Custeio" e "Defesa" e os indicadores do Fator Necessidades de Aperfeiçoamento são "Ensino" e "Pesquisa". Os erros de mensuração das variáveis endógenas foram inseridos na modelagem. Este é o modelo construído com base em uma teoria sobre o fenômeno estudado e sobre a qual se irá testar a plausibilidade. 
Tabela 7

Resumo das Variáveis Especificadas

Observed, endogenous variables:

Custeio
Defesa
Ensino
Pesquisa
Unobserved, exogenous variables:
Fatorl
e1
e2
Fator2
e3
e4

Variable counts

Number of variables in your model: 10 Number of observed variables:

Number of unobserved variables:

Number of exogenous variables:

Number of endogenous variables:

A tabela 7 informa as variáveis especificadas no modelo segundo seu papel na modelagem de equações estruturais. Descreve também uma estatística das variáveis segundo esta natureza. Considerando o diagrama de caminhos do pesquisador, todas as variáveis observadas são definidas como variáveis endógenas do modelo, ao passo que as variáveis latentes e os termos de erro são não observados, participando como variáveis latentes e exógenas do estudo.

Tabela 8

Resumo dos Parâmetros do Modelo

\begin{tabular}{|r|c|c|c|c|c|c|}
\hline & Weights & Covariances & Variances & Means & Intercepts & Total \\
\hline Fixed & 7 & 0 & 0 & 0 & 0 & 7 \\
\hline Labeled & 0 & 0 & 0 & 0 & 0 & 0 \\
\hline Unlabeled & 1 & 1 & 6 & 0 & 0 & 8 \\
\hline Total & 8 & 1 & 6 & 0 & 0 & 15 \\
\hline
\end{tabular}

The model is recursive.

Sample size $=40$

A tabela 8 resume os parâmetros do modelo, indicando o tamanho da amostra, o número de parâmetros que devem ser estimados e os graus de liberdade do modelo. Observe que há 8 pesos de regressão, dos quais 7 são fixos e 1 é estimado. O modelo é recursivo (quando as variáveis não são causas recíprocas umas das outras) e a amostra é constituída de 40 militares. 
Tabela 9

Estimativas das Relações Causais do Modelo

Pesos de Regressão e Variâncias

\section{Pesos de Regressão}

\begin{tabular}{|l|r|r|r|r|}
\hline & Estimate & S.E. & C.R. & P \\
\hline Custeio <--- Fator1 & 1.00 & & & \\
\hline Defesa <--- Fator1 & 1.00 & & & \\
\hline Ensino <--- Fator2 & 1.00 & & & \\
\hline Pesquisa <--- Fator2 & .74 & .20 & 3.64 & $* * *$ \\
\hline
\end{tabular}

Variâncias

\begin{tabular}{|l|c|c|c|c|}
\hline & Estimate & S.E. & C.R. & P \\
\hline Fator1 & 1.08 & .28 & 3.90 & $* * *$ \\
\hline Fator2 & 1.64 & .59 & 2.75 & .01 \\
\hline e1 & .13 & .12 & 1.06 & .29 \\
\hline e2 & .46 & .16 & 2.89 & .00 \\
\hline e3 & .30 & .41 & .73 & .46 \\
\hline e4 & .40 & .24 & 1.66 & .10 \\
\hline
\end{tabular}

Cargas Indicadoras

\begin{tabular}{|l|r|r|r|r|}
\hline & Pesquisa & Ensino & Defesa & Custeio \\
\hline Fator2 & .34 & .61 & -.02 & -.06 \\
\hline Fator1 & -.01 & -.03 & .20 & .70 \\
\hline
\end{tabular}

A tabela 9 indica as estimativas dos pesos de regressão, dos termos de erros em variâncias e a cargas indicadoras. No caso de nosso modelo hipotético, todas as estimativas individuais têm sinais coerentes e erros padrões de magnitudes aceitáveis.

Quanto ao CR, maioria dos pesos de regressão e variâncias de erros é significantes estatisticamente. Portanto, todas as estimativas individuais são estatisticamente consistentes e também exibem robustez teórica. Finalmente, os fatores latentes apresentam cargas significativas nas suas variáveis mais importantes e desprezíveis nas variáveis que não lhes são importantes. 


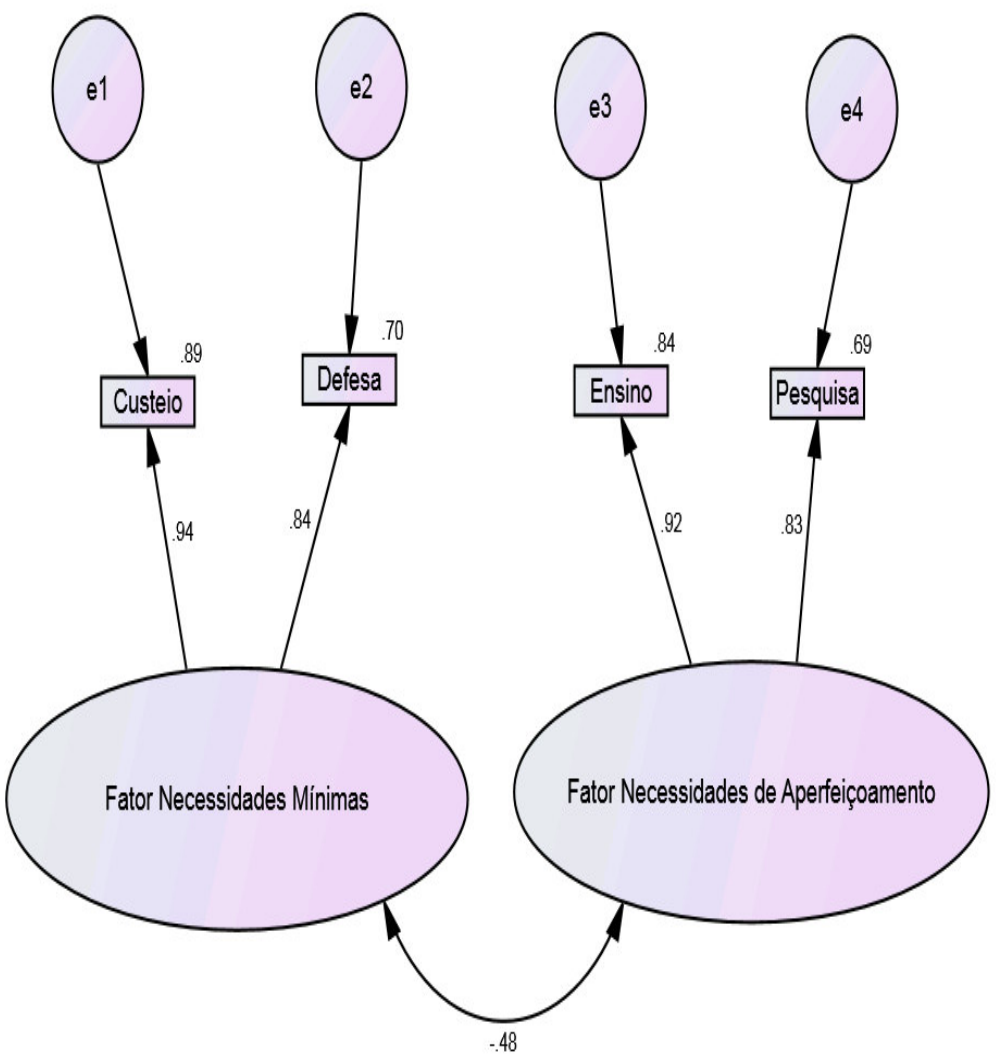

a)Há relevância dos indicadores associados a cada uma das variáveis latentes, uma vez que são muito bem explicados pelas suas respectivas variáveis latentes, apresentando $\beta s>0,7$;

b)A correlação entre os constructos é baixa;

c)Todas as variáveis latentes explicam proporções tolerantes de seus indicadores(coeficientes de explicação $=R^{2} \geq 0,5$ ). 
Tabela 10

Teste do Qui-qudrado( CMIM/DF)

\begin{tabular}{|l|c|c|c|c|c|}
\hline Model & NPAR & CMIN & DF & P & CMIN/DF \\
\hline Default model & 8 & 3.76 & 2 & $\mathbf{. 1 5}$ & $\mathbf{1 . 8 8}$ \\
\hline Saturated model & 10 & .00 & 0 & & \\
\hline Independence model & 4 & 84.46 & 6 & .00 & 14.08 \\
\hline
\end{tabular}

No nosso estudo, o valor-p $=0,15>0,05$, aceita-se a hipótese nula de que as matrizes de dados $(\mathrm{S})$ e estimada $(\Sigma)$ são estatisticamente iguais, o que evidencia bom ajustamento do modelo proposto à base de dados. O CMIM/DF pode confirmar a aceitação de bom ajuste do modelo à realidade. Embora não haja uma regra geral que aponte o valor mínimo aceitável para CMIM/DF, o critério comumente empregado é que essa razão seja menor do que 3. A tabela 10 indica $\mathrm{CMIM} / \mathrm{DF}=1,88<3$, confirmando o bom ajustamento do modelo. A matriz $\Sigma$ é uma estimativa de boa qualidade, isto implica que o modelo hipotético que utilizou-se na análise relaciona realisticamente os constructos "Fator Necessidades Mínimas" e "Fator Necessidades de Aperfeiçoamento" e as percepções dos militares.

Tabela 11

Teste da Bondade do Ajustamento AGFI Índice Ajustado de Qualidade de Ajuste

\begin{tabular}{|l|r|r|r|r|}
\multicolumn{1}{|c}{ A GFI } \\
\hline Model & \multicolumn{1}{|c|}{ RMR } & \multicolumn{1}{c|}{ GFI } & AGFI & PGFI \\
\hline Default model & .04 & $\mathbf{. 9 6}$ & .78 & .19 \\
\hline Saturated model & .00 & 1.00 & & \\
\hline Independence model & .63 & .53 & .21 & .32 \\
\hline
\end{tabular}

É uma extensão do GFI, ajustado através da raiz dos graus de liberdade do modelo proposto pelos graus de liberdade do modelo nulo(todos os parâmetros estruturais são fixados em zero). Valores superiores a 0,90 indicam níveis aceitáveis de ajuste. A tabela 11 indica $\mathrm{AGFI}=0,96>0,90$, o que indica, bom ajustamento dos dados.

Tabela 12

Teste da Bondade do Ajustamento NFI

Índice de Ajuste Normalizado

\begin{tabular}{|l|r|r|r|r|r|}
\hline Model & $\begin{array}{r}\text { NFI } \\
\text { Delta1 }\end{array}$ & $\begin{array}{r}\text { RFI } \\
\text { rho1 }\end{array}$ & $\begin{array}{r}\text { IFI } \\
\text { Delta2 }\end{array}$ & $\begin{array}{r}\text { TLI } \\
\text { rho2 }\end{array}$ & CFI \\
\hline Default model & .96 & .87 & .98 & .93 & .98 \\
\hline Saturated model & 1.00 & & 1.00 & & 1.00 \\
\hline Independence model & .00 & .00 & .00 & .00 & .00 \\
\hline
\end{tabular}

Valores superiores a 0,90 indicam níveis aceitáveis de ajuste. A tabela 12 indica $\mathrm{NFI}=0,96$, o que indica bom ajustamento do modelo. 
Tabela 13

Teste da Bondade do Ajustamento PRATIO

Razão de Parcimônia

\begin{tabular}{|l|r|r|r|}
\hline Model & PRATIO & PNFI & PCFI \\
\hline Default model & $\mathbf{. 3 3}$ & .32 & .33 \\
\hline Saturated model & .00 & .00 & .00 \\
\hline Independence model & 1.00 & .00 & .00 \\
\hline
\end{tabular}

O PRATIO não é um teste de bondade de ajustamento em si, e sim uma medida de parcimônia do modelo. Mas como parcimônia é fator de boa estimação, este teste é empregado como indicador de bondade de ajustamento. Os índices baseados em parcimônia são, usualmente, muito menores do que outras medidas de ajustamento. Valores superiores a 0,60 já são suficientes para considerar o ajuste satisfatório. $\mathrm{O}$ modelo gerado tem um índice de parcimônia PRATIO $=0,33<0,60$, o que indica mau ajustamento. Mas o importante é considerar um balanço geral dos testes de bondade do ajustamento.

Tabela 14

Teste da Bondade do Ajustamento NCP Parâmetros de Não-centralidade

$N C P$

\begin{tabular}{|l|r|r|r|}
\hline Model & NCP & LO 90 & HI 90 \\
\hline Default model & $\mathbf{1 . 7 6}$ & .00 & 11.47 \\
\hline Saturated model & .00 & .00 & .00 \\
\hline Independence model & 78.46 & 52.40 & 111.97 \\
\hline
\end{tabular}

Resulta da tentativa de estatísticos em encontrar uma medida alternativa ao qui-quadrado que fosse menos sensível ao tamanho da amostra. Quanto menor seu valor, melhor, pois isso indica que as matrizes de dados(S) e a estimada $(\Sigma)$ não diferem consideravelmente. A tabela 14 indica $\mathrm{NCP}=1,76$; que é considerado aceitável. Portanto, mais um indicador de bom ajustamento.

Tabela 15

Teste da Bondade do Ajustamento RMSEA Raiz do Erro Médio Quadrático de Aproximação PCLOSE

\begin{tabular}{|l|r|r|r|r|}
\hline RMSEA \\
\hline Model & RMSEA & LO 90 & HI 90 & PCLOSE \\
\hline Default model & .15 & .00 & .38 & $\mathbf{. 1 8}$ \\
\hline Independence model & .58 & .47 & .69 & .00 \\
\hline
\end{tabular}

A RMSEA é uma medida popular de ajustamento, parcialmente em razão de ser um dos índices de ajustamento menos afetado pelo tamanho da amostra, embora para amostras muito pequenas superestima a bondade de ajustamento. A RMSEA avalia quão o modelo se ajusta à matriz de covariância ou correlação da 
população, caso estivesse disponível. Os índices cujos valores sejam inferiores a 0,05 indicam bom ajustamento. Valores que variam de 0,05 a 0,08 são considerados aceitáveis. A tabela 15 especifica RMSEA=0,15; fora dos limites aceitáveis. Para ratificar o resultado, irá se testar a significância desta estimativa pelo PCLOSE. O valor-p associado ao RMSEA, PCLOSE, testa a hipótese nula de que a RMSEA é inferior a 0,05. Se PCLOSE for maior que 0,05; a hipótese nula é aceita, concluindo-se que RMSEA computada é menor que 0,05; indicando bom ajustamento do modelo. A tabela 15 aponta PCLOSE $=0,18$; indicando bom ajustamento do modelo.

\section{Tabela 16 \\ Índices Preditivos de Ajustamento}

AIC
\begin{tabular}{|l|c|c|c|c|}
\hline \multicolumn{1}{|c|}{ Model } & AIC & BCC & BIC & CAIC \\
\hline Default model & $\mathbf{1 9 . 7 6}$ & $\mathbf{2 2 . 1 2}$ & $\mathbf{3 3 . 2 7}$ & $\mathbf{4 1 . 2 7}$ \\
\hline Saturated model & 20.00 & 22.94 & 36.89 & 46.89 \\
\hline Independence model & 92.46 & 93.64 & 99.22 & 103.22 \\
\hline
\end{tabular}

Os Índices Preditivos de Ajustamento avaliam a extensão em que o modelo hipotético será validado em amostras futuras, de mesmo tamanho, extraídas da mesma população da qual foi extraída a amostra original do pesquisador. Os índices preditivos disponíveis no AMOS são: Critério de Informação Akaike (AIC), Critério de Informação BAYES (BIC) e Critério de Informação Akaike Consistente (CAIC). Para todo esse conjunto de índices, há comparação entre modelos, sendo considerado de melhor ajustamento os que exibirem os menores valores de $A I C, B I C$ e $C A I C$. Na tabela 16, o modelo default, que comparado com os outros modelos, apresenta os menores índices preditivos, indicando validade e confiabilidade dos resultados da modelagem.

\section{Tabela 17}

\section{Índice de Bondade de Ajustamento ECVI} Índices de Validação Cruzada Esperada

\begin{tabular}{|l|c|c|c|c|}
\hline Model & ECVI & LO 90 & HI 90 & MECVI \\
\hline Default model & $\mathbf{. 5 1}$ & $\mathbf{. 4 6}$ & $\mathbf{. 7 6}$ & $\mathbf{. 5 7}$ \\
\hline Saturated model & .51 & .51 & .51 & .59 \\
\hline Independence model & 2.37 & 1.70 & 3.23 & 2.40 \\
\hline
\end{tabular}

Os Índice de Validação Cruzada Esperada são uma aproximação da qualidade de ajuste que o modelo estimado apresentaria em outra amostra de dados, de igual tamanho. Não há intervalos especificados para valores aceitáveis, sendo mais bem utilizados na comparação com modelos alternativos. Comparando com os modelos alternativos, apresenta em sua maior parte os menores índices, indicando boa validade e confiabilidade. 


\section{Tabela 18 \\ Índice de Bondade de Ajustamento(HOELTER)}

\section{HOELTER}

\begin{tabular}{|c|c|c|}
\hline Model & $\begin{array}{c}\text { HOELTER } \\
\mathbf{. 0 5}\end{array}$ & $\begin{array}{c}\text { HOELTER } \\
\mathbf{. 0 1}\end{array}$ \\
\hline Default model & $\mathbf{6 3}$ & 96 \\
\hline Independence model & 6 & 8 \\
\hline
\end{tabular}

O Índice de Ajustamento de HOELTER ou N Crítico indica o maior número para o tamanho da amostra para que se confiem nos resultados do Qui-qudrado. O AMOS fornece o $\mathrm{N}$ crítico nos níveis de significância de $5 \%$ e $1 \%$. Observando a tabela $18, \mathrm{~N}$ crítico $=63$, ao nível de $5 \%$ de significância, verificamos que para o teste do quiquadrado fosse considerado confiável seria necessária uma amostra de no máximo até 63 militares. A base de dados deste estudo tem 40 profissionais das armas, logo o CMIM é confiável.

Diante do estudo realizado, pode-se concluir que o modelo proposto na análise de modelagem de equações estruturais tem bom ajustamento e este modelo surgiu da análise fatorial exploratória, o que ratifica os constructos formulados e as suas respectivas variáveis observadas discriminantes.

\section{5-CONCLUSÃO}

O objetivo deste estudo foi realizar a modelagem de equações estruturais, através do software estatístico AMOS, para identificar os fatores que essencialmente refletem o que os militares avaliam como carentes de recursos na área da defesa.

A formalização da teoria a cerca das relações causais se fundamentou nos resultados de uma análise fatorial exploratória, envolvendo as medidas de percepções coletadas em uma pesquisa por amostragem por quotas realizada em áreas militares de grande movimento.

A análise fatorial exploratória permitiu tecer a teoria de que as medidas de percepção(custeio, defesa, ensino e pesquisa) de falta de recursos de militares se movem para dois traços latentes: "Fator Necessidades Mínimas" e "Fator Necessidade de Aperfeiçoamento". Estes novos eixos temáticos de percepção respondem por $89 \%$ da variação total do espaço de partida das percepções de militares quanto à defasagem de recursos dirigidos às Forças Armadas.

A modelagem de equações estruturais, em seu balanço geral, atestou a boa aderência dos dados à realidade populacional e ratificou significância das forças das relações causais estimadas.

$\mathrm{O}$ estudo visou buscar apoio dos militares ativos, que vivenciam a realidade militar em seu dia a dia, aos argumentos do Ministério da Defesa de que o orçamento é insuficiente para as necessidades mínimas do setor. Com a consecução dos resultados, o referido apoio pode ser considerado pertinente e o objetivo do estudo alcançado. 
Acredita-se que o mérito deste estudo foi procurar produzir conhecimento ou informação que pudesse cientificamente fundamentar as argumentações do Ministério da Defesa de que as Forças Armadas precisam de maiores recursos para o bem promover a defesa e a proteção das fronteiras de nosso país e assim garantir sempre a soberania nacional.

A investigação é meramente preliminar, o ideal seria uma amostra com maior representatividade, mas abre caminhos para estudos mais generalizáveis sobre a temática estudada.

\section{6-REFERÊNCIAS BIBLIOGRÁFICAS}

[1]BABBIE, Earl. Métodos de pesquisas de survey. Belo Horizonte:Ed. UFMG, 1999.

[2]BEDEIAN, A.G.; DAY, D.V.; KELLOWAY, E.K. Correcting for measurement error attenuation in structural equation models: some important reminders. Educational and Psychological Measurement, [S.1.], v. 57, n. 5, p. 785-799,c1997. Copyright Holder: Sage Publications, Inc.

[3]BENTLER, Peter M.; WU, Eric J.C. EQS 6 for windowsuser's guide. Encino: Multivariate Software Inc., 2002.BOLLEN, Kenneth A. Structural equations with latent variables. New York: John Wiley \& Sons, Inc., 1989.

[4]BENTLER, Peter M.; WU, Eric J.C. EQS 6 for windows user's guide. Encino: Multivariate Software Inc., 2002. BOLLEN, Kenneth A. Structural equations with latent variables. New York: John Wiley \& Sons, Inc., 1989. Associates, Inc.

[5]BOURDIEU, Pierre. O poder simbólico. Rio de Janeiro: Bertrand Brasil, 1998.

[6]BYRNE, Barbara M. Structural equation modeling with EQS and EQS/windows: basic concepts, applications and programming. Thousand Oaks: Sage Publications, 1994.

[7]DUNCAN, Otis Dudley. Path analysis: sociological examples. In: BLALOCK JR, H.M. (Org). Causal models in the social sciences. Chicago: Aldine Publishing Company,1971. p. 115-138.

[8]EDWARDS, J. R.; BAGOZZI, R. P. On the nature and direction of relationships between constructs and measures. Pyschological Methods, Washington, v. 5, n. 2,p. 155-174, c2000. Copyright Holder: American Psychological Association.

[9]FINCHAM, F.D. et al. Marital satisfaction and depression: Different causal relationships for men and women? Psychological Science, Washington, v. 8, n. 5, p. 351-357,c1997. Copyright Holder: American Psychological Association.

[10]HOX, J. J.; BECHGER, T. M. An Introduction to structural equation modeling. Family Science Review, Minneapolis,v. 11, p. 354-373, 1998.

[11]HOYLE, R. H.; SMITH, G. T. Formulating clinical reserach hypotheses as structural equation models: A conceptual overview. Journal of Consulting and Clinical Psychology, Washington, v. 62, n. 3, p. 429-440, c1994. Copyright Holder: American Psychological Association.

[12]KAPLAN, David. Structural equation modeling:foundations and extensions. Thousand Oaks: Sage Publications, 2000.

[13]KENNY, D. A.; KASHY, D. A.; BOLGER, N. Data analysis in social psychology. In: GILBERT, D.; FISKE, E.;LINDZEY, G. (Orgs.) Handbook of social psychology.Boston: McGraw-Hill, 1998. v. 1, p. 252-259.

[14]KLEM, L. Structural equation modeling. In: GRIMM, L.G.; YARNOLD, P.R. (Eds.). Reading and understanding more multivariate statistics. Washington,D.C.: Copyright Holder: American Psychological Association, c2000.

[15]KLINE, Rex B. Principles and practice of structural equation modeling. New York: The Guilford Press, 1998.

[16]MACCALLUM, R. C. et al. The problem of equivalent models in applications of covariance structure analysis.Psychological Bulletin, Washington, v. 11, n. 1, p. 184-199, c1993. Copyright Holder: American Psychological Association.

[17]MARSHALL, G. N.; LANG, E. L. Optimism, self-mastery,and symptoms of depression in women professionals.Journal of Personality and Social Psychology, 
Washington,v.59, n.1, p.132-139, c1990. Copyright Holder:American Psychological Association.

[18]MCDONALD, R.P.; RINGO HO.; MOON-HO. Principles and practice in reporting structural equation analyses.Psychological Methods, Washington D.C, v. 7, n. 1, p. 64-82, c2002. Copyright Holder: American Psychological Association.

[19]RAYKOV, T., TOMER, A.; NESSELROADE, J. R. Reporting structural equation modeling results in psychology and aging: some proposed guidelines. Psychology and Aging, Washington, v. 6, n. 4, p. 499-503, c1991. Copyright Holder: American Psychological Association .

[20]SLIWIANY, Regina Maria. Sociometria: como avaliar a qualidade de vida e projetos sociais. Petrópolis: Vozes, 1997.

[21]TOMAS, Jose M.; OLIVER, Amparo. Rosenberg's selfesteem scale: two factors or method effects. Structural Equation Modeling, [S.1.], v. 6, n. 1, p. 84-98, c1999. Copyright Holder: Lawrence Erlbaum Associates, Inc.

[22]TURNER, Malcom E.; STEVENS, Charles D. The regression analysis of causal paths. In: BLALOCK JR, H.M. (Org). Causal models in the social sciences. Chicago: Aldine Publishing Company, 1971. p. 75-100. 